\title{
A CNS guard as prickly as a hedgehog
}

Compromised function of the blood-brain barrier (BBB) and entry of activated lymphocytes into the central nervous system (CNS) are associated with the pathogenesis of neuroinflammatory diseases, such as multiple sclerosis. A recent study in Science indicates that Hedgehog signalling promotes $\mathrm{BBB}$ integrity and contributes to the immune-privileged state of the CNS.

Binding of sonic hedgehog $(\mathrm{SHH})$ to the receptor Patched 1 (PTCH1) activates Smoothened (SMO) and initiates gene transcription under the control of the GLI family of transcription factors. Alvarez et al. detected $\mathrm{SHH}$ expression in astrocytes, active $\mathrm{SHH}$ protein in the supernatants of astrocyte cultures, and PTCH1 and SMO expression in BBB endothelial cells. Thus, they hypothesized that Hedgehog signalling might regulate BBB function.

Indeed, both the paracellular permeability and the intercellular transport activity of human BBB endothelial cells were found to decrease in the presence of $\mathrm{SHH}$ or a PTCH1 agonist, as Hedgehog signalling induced the expression of tight junction proteins. Moreover, BBB leakage was observed in mice that were treated with a SMO antagonist or that lacked SMO expression in endothelial cells. Thus, abrogation of the Hedgehog pathway in BBB endothelial cells can perturb their function.

In addition to promoting the formation of tight junctions between BBB endothelial cells, Hedgehog signalling was found to prevent the chemoattraction of leukocytes to the CNS by decreasing the expression of chemokines and adhesion molecules by BBB endothelial cells. Moreover, Hedgehog signalling was shown to regulate the adhesion properties and effector functions of $\mathrm{CD}^{+} \mathrm{T}$ cells (which also express PTCH1). When polarized in vitro in the presence of $\mathrm{SHH}$, T helper $17\left(\mathrm{~T}_{\mathrm{H}} 17\right)$ cells displayed lower levels of CD6 (which has been linked with susceptibility to multiple sclerosis) and $\mathrm{T}_{\mathrm{H}} 1$ cells produced less interferon- $\gamma($ IFN $\gamma)$, whereas $\mathrm{T}_{\mathrm{H}} 2$ cells expressed higher levels of adhesion molecules.

Finally, mice with experimental autoimmune encephalomyelitis that were treated with a Hedgehog antagonist displayed higher numbers of CNS-infiltrating leukocytes and increased disease severity compared with untreated mice. The finding that pro-inflammatory cytokines (such as tumour necrosis factor and IFN $\gamma$ ) induce $\mathrm{SHH}$ expression by astrocytes indicates that $\mathrm{SHH}$ has a compensatory role in the CNS during neuroinflammation.

Maria Papatriantafyllou

ORIGINAL RESEARCH PAPER Alvarez, J. I. et al. The hedgehog pathway promotes blood-brain barrier integrity and CNS immune quiescence.

Science 1 Dec 2011 (doi:10.1126/science.1206936) 